\title{
Improvisation as Tool and Intention: Organizational Practices in Laptop Orchestras and Their Effect on Personal Musical Approaches
}

\section{J eff Albert}

\section{Introduction}

Laptop orchestras have developed out of musical situations rooted in the Western European classical tradition and its experimental fringe. These groups are often based in academic institutions and populated by students and faculty. Improvisation is a common practice in laptop orchestras, as in many other ensembles in the experimentalist tradition. This article explores the different roles that improvisation plays in the development and performance of music for laptop orchestras and attempts to show how this practice has affected the musical outlook of some members of these ensembles. $^{1}$

Using the repertoire of the Laptop Orchestra of Louisiana (of which I was a member) as a starting point, I present a focused analysis of some of the practices of academic laptop orchestras, and of how participation in these ensembles can provide students with a gateway to improvisation. The "LOLs" has nine members performing from five computer stations, and often also uses acoustic instruments. ${ }^{2}$ It was established in 2009 and is made up of graduate students and faculty at Louisiana State University in Baton Rouge. All but one of its members have been affiliated with the School of Music, and most performers also compose for the group.

Their improvisational practices will be presented in terms of two possibly overlapping domains: improvisation as a tool, and improvisation as intent. When improvisation acts as a tool for exploring new instrument designs or compositional structures, ensemble participants can improvise in settings that are free from traditional instruments and their accompanying pressures and expectations. Introducing improvisational practices in the context of unfamiliar laptop-based instruments can often encourage musicians to incorporate improvisation into their musical practice on their traditional instruments.

\section{Definitions}

Bruce Ellis Benson delineates the difference between performance and improvisation: "A performance is essentially an interpretation of something that already exists, whereas improvisation presents us with something that only comes into being in the moment of presentation" (25). Consider also Daniel Trueman's definition of a laptop orchestra: an "ensemble of computer-based musical meta-instruments." The laptop is referred to as a meta-instrument because its instrumental properties change from piece to piece, and sometimes within pieces. Whereas a violin remains a violin in a concert from start to finish, a laptop in a laptop orchestra will take on many different instrumental guises.

These instruments, or meta-instruments to use Trueman's term, fall into two broad categories. The first is what I call "direct sound control" instruments, where there exists a direct temporal connection between the actions of the performer and the creation of sound. The second category includes instruments that are process- or code-controlled. These are instruments in which the performer launches a process, or sometimes writes the code during the performance (known as live coding), that then takes over the lower-level control of making the sounds. This removes the temporal immediacy from the performer's control, but creates a scenario in which musical textures of much greater complexity can be created.

Providing a singular description of the sound of laptop orchestras can be difficult. A wide variety of sounds are possible, including classic electronic music sounds such as beeps, squeaks, clicks and swishes, as well as classic acoustic instrumental sounds, and electronic manipulations of those sounds. The textures can be static, ambient, or densely rhythmic. The music can be melodic or abstract and dissonant, and the timbres can be just as varied. It is also common practice for new software instruments to be designed for each presentation. A symphony orchestra can play Mozart, Ravel, and Ligeti pieces and achieve a similar variety of style and texture; but a symphony orchestra maintains the timbral qualities of its constituent instruments. In a laptop orchestra, each meta-instrument (laptop) has unlimited timbral and musical possibilities. 


\section{Domains of Improvisation}

Improvisation as intent is the domain in which the act of improvising is the motivating factor. These are musical events driven by improvisational activity, and the goal of performers is to find new or compelling expressions and modes of interaction (Belgrad 2). This practice comes from an improvisational aesthetic lineage shared by experimental musicians working with both acoustic and electronic instruments. Free Improvisation is a classic example of improvisation as intent.

Improvisation as a tool is the domain that uses improvisation as a way to explore new instruments and modes of operation. A majority of the improvisation that happens in laptop orchestras and ensembles falls into this category: a tool for discovering the possibilities of new instruments or ideas, but a tool that also often leads to an agreed-upon shape or structure whereby the initial improvisatory nature settles into standardized practice (Wolek).

The primary difference between these two domains is how much improvisation survives the journey from rehearsal to performance. Where improvisation as intention survives as the goal of performance, improvisation as tool often settles into memorized content through the course of rehearsals. Nathan Wolek of Stetson University describes how improvisation as tool is practiced in his group MPG: "When we first develop a piece, it is usually a complete improvisation. Through repetition, we eventually find a 'usual way' of performing the piece and a structure develops." The idea of a pop/rock style evolutionary cycle in which improvised explorations settle into established and repeatable ideas is also presented by Scott Hewitt and the HELO ensemble of Huddersfield University: "Improvisation has proven itself to be a good technique at the start of the year [to] highlight control problems in the first weeks. Often improvisational ideas are developed into pieces."

These two domains can overlap, and they often do. According to Jesse Allison from the LOLs, "It's generally through improv that [the composers] come to the sounds, effects, textures, and things that they use in the piece; e.g. they build an instrument, then play with it to get a feel for what it can do. Then they compose-either improv, semistructured, or notated." Wolek supports this idea of overlap: "The piece is never completely notated and players maintain latitude to improvise within the structure."

\section{Repertoire of the Laptop Orchestra of Louisiana, and the Role of Improvisation in Each Piece}

Five pieces from the spring 2011 repertoire of the Laptop Orchestra of Louisiana will serve as the basis for an examination of improvisational practices within these domains. Video of performances of these and other pieces is available on the LSU Experimental Music \& Digital Media YouTube channel:

http://www.youtube.com/user/lsuEMDM/videos. Input from the leaders or conductors of five other groups in the US and Europe will show that the LOLs's practices are not singular, but are comparable to the practices of similarly situated laptop orchestras.

Improvisations and Transformations is a fully improvised "piece" performed by the LOLs since 2009. The aspects of the piece that remain consistent from performance to performance are technical and conceptual in nature, but the musical characteristics can vary wildly. It always features some acoustic instrumentalists, each matched with a laptop performer using the GUA, a live sampling and manipulation instrument designed by Nick Hwang for use in improvisations. This piece is meant to showcase some of the strong acoustic performers who are members of the LOLs and to force our instrument design into a place that privileges subtle real time control and performance flexibility. The acoustic instrumentalists perform as they would in any other free improvisation setting. The musicians control their GUA instruments from an iPad interface and get visual feedback from the laptop screen. The GUA samples live input and manipulates it in terms of pitch, speed, and direction. It also has the ability to apply digital effects to the acoustic performance.

Improvisations and Transformations is a case of improvisation as intent, but improvisation is also a tool in terms of the design of the GUA. Instead of using improvisation to explore what the instrument could do and then composing for those capabilities, improvisation was used to push the instrument to find the things it couldn't do: musical experience 
led to the desire to add new functionalities. GUA is a very versatile instrument because of the improvised musical settings in which it was developed. Now that it has reached a level of maturity as an instrument, it has also come to be used in other, more structured, musical settings,

Forbidden Butch is a piece I composed in the fall of 2010, the product of my desire to create an instrument that uses the joystick controllers in the LOLs equipment collection. The joystick's $x$ - and y-axis each control the frequency of an oscillator in an FM synthesis configuration. Buttons on the joystick controller set the time and feedback of a digital delay.

Originally, I planned to devise a notation system for this instrument that would allow me to compose a fully notated piece, something lacking in the LOLs's repertoire. I am an improviser at heart and enjoy establishing musical spaces from which to improvise, but in this case I wished to push beyond my comfort zone. Of course, creating notated music requires a system of notation that will suit the instruments for which one is composing. Since this joystick instrument has no real tempered pitch reference, standard notation was not a very rewarding way to record the composition.

I went through a few different paradigms of test notation, but never did find a system that felt intuitive to both the composer and performer. I tried a couple of different graphic notations that followed the idea of drawing a shape to be recreated on the joystick. These ideas were fairly successful at communicating pitch shapes, but much less successful at communicating time components.

While working on this piece, I had a chance to hear Butch Morris, who produces conducted improvisations (Conductions $\left.{ }^{\mathrm{TM}}\right)^{3}$ in which he communicates musical organization through an elaborate system of physical gestures. Specific musical elements are usually improvised by the performers, but the overall shape and form is rigidly controlled by Mr. Morris. It later struck me that conducted improvisation would be a great means of organizing my piece, as it gives a coherent organization to the overall musical experience while allowing individual performers to improvise lower-level details—such as specific choices of pitch and rhythm—that might otherwise have been notated. ${ }^{4}$

The joystick controller provides musicians with a "safe" medium through which to engage in improvisation. Take a hypothetical example of an ensemble member who is an accomplished violinist. She has excellent technique, reads music well, and performs composed music from memory-but is uncomfortable improvising on her violin, especially in front of others. Perhaps she has a reputation as a skilled violinist that she must protect; however, as she has no reputation on joystick instruments, she may feel freer to explore improvised practices in this environment. The format of Forbidden Butch may also be comforting to new improvisers, as the individual is not expected to generate all the musical ideas, but simply to realize gestures put forth by the conductor. Here, improvisation is still an intention, but also functions as a tool to fill in gaps of musical detail that are difficult to communicate or notate. ${ }^{5}$

5 Screaming Amps for Any Number of Electronic Instruments was composed by J. Corey Knoll and first performed by the LOLs. Mr. Knoll is a PhD candidate in Music Composition at LSU and a founding member of the LOLs. The piece is part of his " 5 Screaming X" series that began with 5 Screaming Composers. Almost all of the lower-level musical activity is improvised and the piece may be performed by both pitched and non-pitched instruments.

The musical predecessor to "5 Screaming Amps" was a group activity called "own instrument improv." Each member of the LOLs builds/programs a laptop-based instrument. These instruments are as individual as their designers, with a wide variety of controller styles and an equally wide palette of possible sounds. The control methods for these instruments range from joysticks and MIDI pads to Wii video game controllers, a didgeridoo, and a decorative holiday tin with a contact microphone. The "own instrument improv" is a freely improvised musical practice that began as a way to explore these new instruments while also developing musical-collaboration skills. We eventually performed the "own instrument improv" at an afternoon outdoor concert on campus.

As we continued to do the "own instrument improv," we began to discuss possible musical shapes, or "density maps," before we began to play. " 5 Screaming Amps" is Corey's codification of these musical paths and an example of the group's preference for some degree of structure. The composition consists of a graphic score (see Fig. 1), which details an approximately seven-minute structure in terms of musical density and gestural construction, and two pages 
of verbal instructions outlining the logistics of the performance and explaining the graphic score. Instructions may include "fast driving rhythmic activity," "loud bursts," and "long lyric melodies" (Knoll).

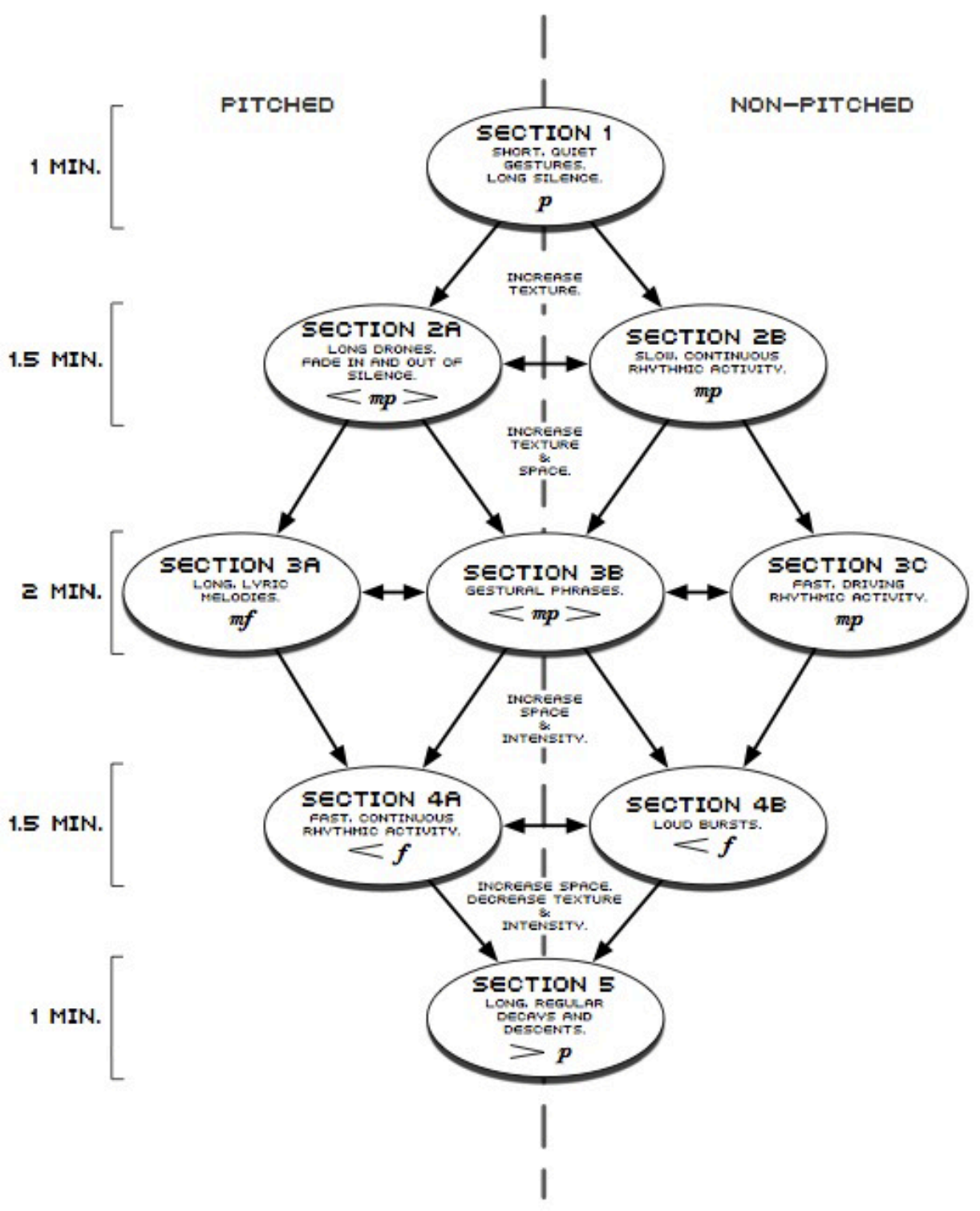

Figure 1. J. Corey Knoll, excerpt from 5 Screaming Amps for Any Number of Electronic Instruments graphic score. Watch and listen to "5 Screaming Amps" at http://www.youtube.com/watch?v=uV0muG0-DrY. 
Stephen David Beck composed Concerto Grosso for shofar, conch shell, and four laptops using the GUA. Dr. Beck is the Derryl and Helen Haymon Professor of Music at LSU and the founder and conductor of the LOLs. This piece is another example of improvisation filling in notational inadequacies, in this case for instruments unable to accurately perform from standard notation. The notation consists of high-level instructions for each player, laid out in score form on a timeline.

Concerto Grosso [sic] was a challenge for me on several levels. First was the notational issue of how does one notate for each (shofar, conch, iPad controlled gua). Rather than try and develop some new notational system that each of us would have to learn (read highly-probable-point-of-failure), I felt that simple text descriptors of what I wanted to have happen would be sufficient. On the other hand, I felt that I didn't want to impose too much specificity in the notation as I wanted the piece to feel organic as it evolved. That said, I would not describe the piece conceptually wholly as an improvisation. My goal for the piece was finding a way to create structured control over the gua players, and provide some quasi-notational guidance for the shofar/conch players. The improvisation was in part a short hand for addressing the local content while using the notation to create the larger and broader structures. (Beck)

In Concerto Grosso, the role of improvisation falls squarely into both domains. The piece would be exceedingly difficult to notate or communicate without improvisation, and even if it could be completely prescribed, the spirit of improvisation is part of the composer's intention.

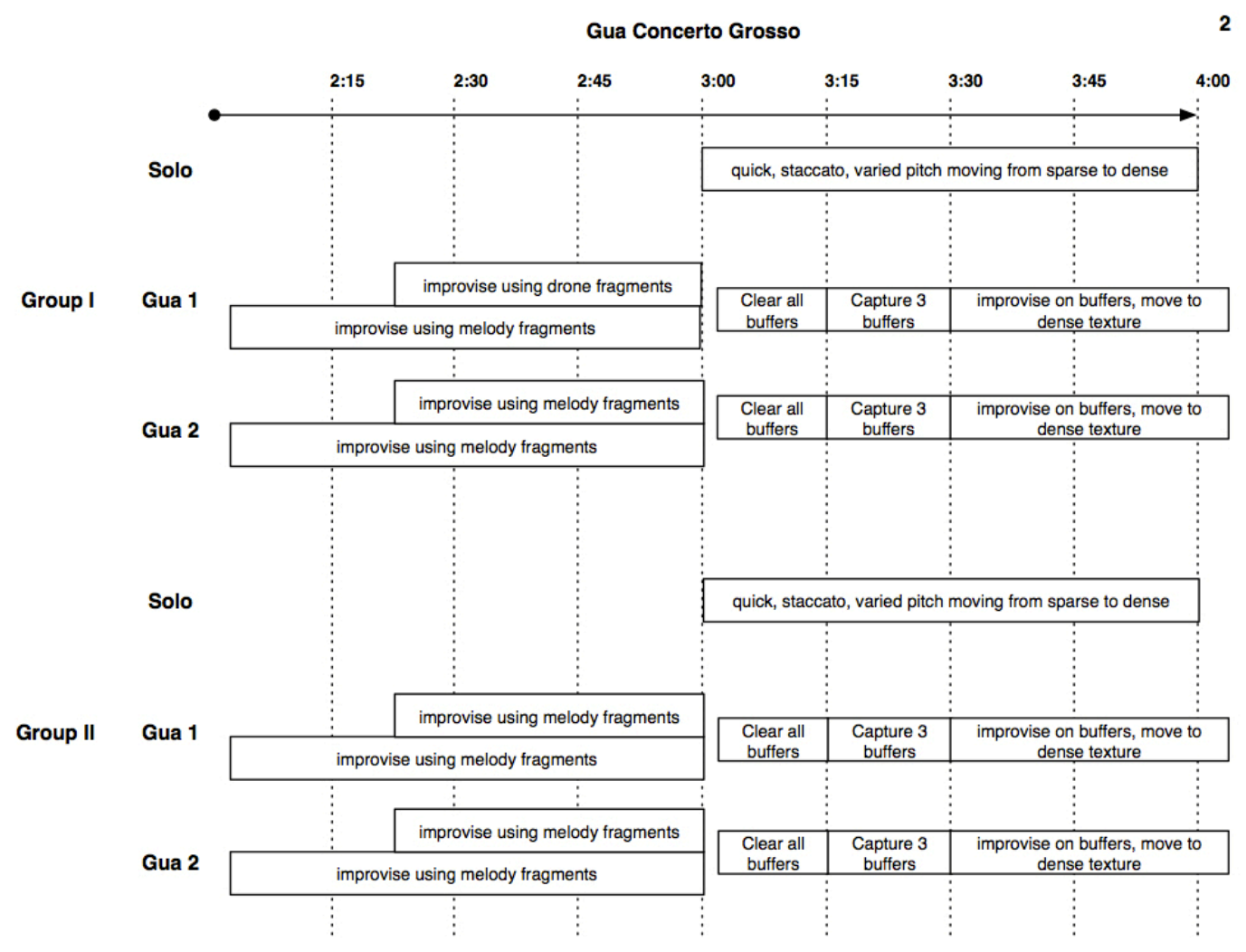

Figure 2. Stephen David Beck, Concerto Grosso excerpt. This excerpt happens from approximately 2:20-4:20 of this video: http://www.youtube.com/watch?v=2QiSFFcWuU8. 
WTFreq (pronounced "What the Freak") is a composition by Nick Hwang, a PhD candidate in Music Composition at LSU and a founding member of the LOLs. This piece includes an instrument controlled by Wiimotes (game controllers from the Wii video game console) and instructions in the form of text and pictures that appear on each performer's laptop. When I asked Nick if he would have fully notated it, he responded,

Yes, if I could have fully notated all of it, I would have. Because of time constraints, I feel the written text would facilitate the musical-instruction-giving. AND allowed for simultaneous INDIVIDUAL instruction. This allowed for different things to occur, without having a 'conductor' give each performer instruction. BUT I purposely chose open-ended improvisatory instructions, to encourage an inter-performer interaction, such as "SHAKER: create counterpoint with Performer 4" and "With the LEADER....", etc. [. . .] I don't feel Improvisation for WTFreq was a 'solution' to deficiencies in notation. YES: there are few wii-mote pieces out in the world, but there isn't a standardized notation practice for it yet. I feel like using common game control instructions (like you would see in a game manual) could be an effective and easily translatable mode of notation. [. . .] If I were to fully notate it, though, it would have a different feel to it. [. . .] Mainly, with musicians who are familiar with their instrument and with each other, the sense of improv imbues a more organic performance, compared to a fully-noted score which may seem more rigid.

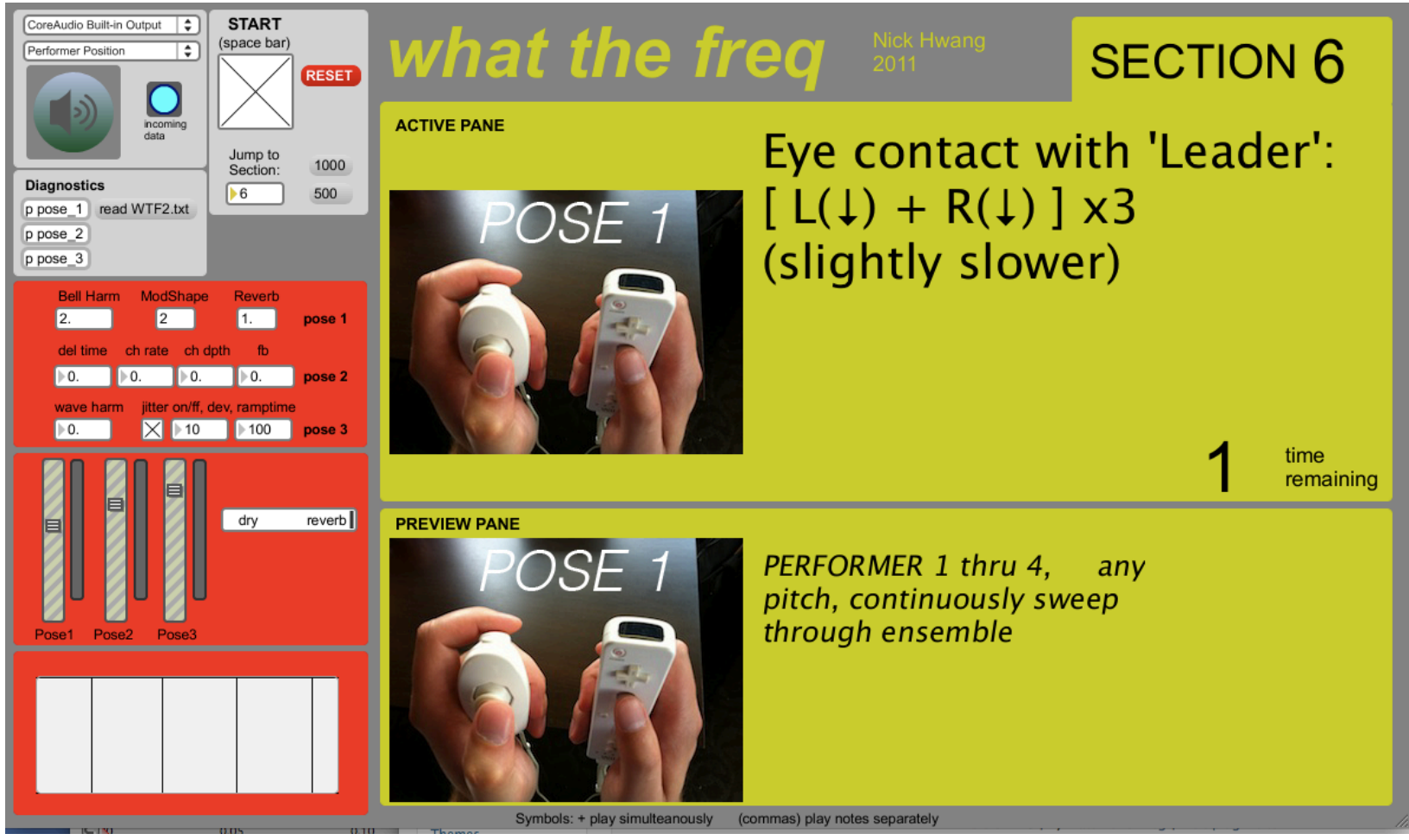

Figure 3. An image from the on-screen instructions to Nick Hwang's WTFreq. Watch and listen to "WTFreq" at http://www.youtube.com/watch?v=eBGf3ly9Ye8.

WTFreq falls into the domain of improvisation as tool based on how much improvisation survives from rehearsal to performance. Early rehearsals varied widely, but as the ensemble learned the piece and became comfortable with the Wiimote, outcomes became more uniform and consistent. We found the "usual way" to perform the piece through improvisation, maintaining the organic performance that Hwang desired in combination with a consistent performance practice that preserved the identity of the piece.

The use of familiar game controllers and game control instructions in WTFreq creates an aspect of comfort and familiarity for performers. Most members of the orchestra had played with a Wii, so the physical interface was familiar. One may hear a novice musical improviser say, "I don't know how to do this," but we all feel like we know how to play 
video games. As with Forbidden Butch, moving musical improvisation from a primary instrument to an instrument where there is no expectation of virtuosity_in this case a familiar interface-allows the novice improviser to shed some inhibitions and generate confidence.

\section{Effect on Performers}

Laptop orchestras have members of varied backgrounds, often with more formal computer science training than music training, and it is not uncommon for laptop orchestra courses to be cross-listed in music and computer science. In my experience, however, it is also common to have performers who are experienced on other traditional instruments, who feel completely at home on stage reading from printed music or recreating music from memory. The interfaces of laptop music provide an ideal environment for easing such musicians into improvised practices. Improvising on joysticks, Wii-motes, or any other interface besides their primary instruments allows performers to explore their musical instincts without risking their reputations. No one expects virtuosity from a joystick performance, although that may change in the near future. Laptop orchestras are not the only ensembles that excel at the exploration of improvisation-many modern chamber ensembles and free improvisation groups can also encourage such exploration-but their use of so many novel interfaces in improvisational settings makes them remarkably well suited to this process.

Jhayson Pathak was a member of MPG (Mobile Performance Group, the Stetson laptop ensemble) while an undergraduate student at Stetson University in DeLand, Florida. I asked Jhayson how improvising in the context of a laptop orchestra affected his musical and compositional practice:

I think the greatest thing was taking away the fear of improvising. I double majored at University and the classical guitar is a totally different monster. Human error so delicate that it terrified me when I played in front of people. Now you always have computer errors but you're not alone. You have people next to you who have their roles to do while you get your head on straight or fix your Max Patch. That's always fun. [. . .] I can't really consider a piece, say a guitar quartet piece that I write, as something strictly non-improvised. I have to play around with an idea; mold it, look at it in different light to find where it shines brightest. I can't do that without improvising. So even though I end up with a piece for a classical guitar quartet, sans improvisation, I've still used it to write the piece.

Susannah Montandon is a graduate student studying cello at LSU. She came to our group with a great deal of curiosity about computer music, but very little experience beyond her significant expertise as a performing cellist. Susannah was actually asked to improvise on her cello as part of a performance of Improvisations and Transformations, and she also improvised on computer instruments during other pieces. I asked Susannah if improvising in a laptop orchestra changed her opinion about the utility or validity of improvisation:

I used to think that I needed to learn how to improvise to actually make it successful. To a certain degree that might be true, but also one might not necessarily need to know all styles and techniques associated with improvising. Sometimes you just have to start playing and see where the music leads you. Those initial first few notes are sometimes the hardest to overcome-similar to inertia. Now I am not as hesitant to improvise. I will even improvise before a practice session to warm up. Sometimes I will also use improvisation to test certain characteristics the composer may or may not have put within the music to create an atmosphere about the composition. It's not always that successful, but it's all part of the learning process. I also use improv during my practice sessions to help my hands and body relax when I realize any tension. I would say that improvisation has turned into a useful tool and also a musical practice.

I also asked Susannah if she now considered herself an improviser:

I definitely did not consider myself an improviser before LOLs. I would not necessarily consider myself with the title of "improviser" now just because I have had some experience with it. I would say that I am more comfortable with the idea of improvising and have assimilated it into my current musical practice. I still find the task of "doing" before an audience to be quite challenging. 
These are just two examples of students exposed to improvised music through academic laptop orchestras. Each student affirms that adding improvisation to their personal musical practice has benefited their overall musicianship. In each example, the musician adopted practices that fall firmly into the domain of improvisation as a tool. These students did not start performing on their primary instruments in improvised situations, but they did each incorporate improvisation into their regular routines for practice or composition. I do not propose that experiences with new computer-based instruments and novel controllers will turn conservatory-trained musicians into intentional improvisers, but as in the cases of Jhayson and Susannah, participation in a laptop orchestra can enrich and expand a musician's creative horizons.

\section{Conclusion}

It is unlikely that the founders of most academic laptop orchestras envisioned (re)introducing improvised musical practices to academic music. The reality of emerging technologies and the performance practices they engender, however, is that improvisation is bound to be part of the scenario. The first music produced on any new instrument is necessarily improvised in order to explore its possibilities-only later are we able to conceive of specific compositions or techniques. Laptop orchestras often invent new instruments for each new piece, so improvisation is bound to be part of that developmental process.

These five pieces from the Laptop Orchestra of Louisiana illustrate how varied an orchestra's improvisational practices can be and what roles those practices play in the development and performance of the orchestra's music. Improvisation can function as a tool to help discover musical essence or facilitate its performance, or it can be the essence itself. In either case, members gain valuable insight into improvised musical practices, perhaps the most lasting heritage of this early generation of academic laptop orchestras.

\section{Acknowledgments}

I'd like to thank Dr. Stephen David Beck and Dr. Jesse Allison, the LSU faculty members responsible for the Laptop Orchestra of Louisiana, for their support and encouragement. I'd also like to thank Dr. Brett Boutwell of LSU for his invaluable advice at a crucial point in this project. Special thanks go to Susannah Montandon, Jhayson Pathek, Nick Hwang, J. Corey Knoll, and Brandon Thomas-and to the laptop orchestra leaders who took the time to communicate with me: Dr. Ge Wang of Stanford University, Patrick Borgeat of Benoit and the Mandelbrots, Dr. Nathan Wolek of Stetson University, Scott Hewitt of Huddersfield University, and Dr. Rebecca Fiebrink of Princeton University.

\section{Notes}

${ }^{1}$ The terms "laptop orchestra" and "laptop ensemble" are often used interchangeably. The word "orchestra" will be used in this paper, although some groups use the term "ensemble" with equal efficacy.

${ }^{2}$ Of the respondents to an email survey I sent to leaders or conductors of six laptop orchestras in the US and Europe, five groups had between four and ten members, plus one with twenty members.

3 http://www.conduction.us/

${ }^{4}$ The idea of conducted improvisation is common in laptop orchestras and is used on another LOLs piece by Lindsey Hartman called InTwerp, as well as in the Princeton Laptop Orchestra (PLOrk) version of Ge Wang's Clix.

${ }^{5}$ I should note that this piece was structured in this way for musical and logistical reasons; the creation of a safe environment for novice improvisers was simply a happy byproduct.

${ }^{6}$ Each of the email survey respondents acknowledged using broad musical instructions to guide or organize their improvisations. This type of verbal instruction is probably the most common way of structuring pieces governed by improvisation as intent. 


\section{Works Cited}

Albert, Jeff. "Forbidden Butch." 2011. TS, Max/MSP file.

Allison, Jesse. "Improvising Laptop Ensemble Survey." Message to the author. 9 April 2011. E-mail.

Beck, Stephen David. Message to the author. 29 May 2011. E-mail.

---. "Concerto Grosso." 2011. TS.

Belgrad, Daniel. The Culture of Spontaneity: Improvisation and the Arts in Postwar America. Chicago: U of Chicago P, 1998. Print.

Benson, Bruce Ellis. The Improvisation of Musical Dialogue: A Phenomenology of Music. New York: Cambridge UP, 2003. Print.

Hewitt, Scott. "Improvising Laptop Ensemble Survey." Message to the author. 13 April 2011. E-mail.

Hwang, Nick. Message to the author. 24 May 2011. E-mail.

---. "Gua: A Live Manipulation and Sampling Digital Instrument for Laptop and Ipad." 13 March 2011. Web. 23 May 2011.

---. "Wtfreq." 2011. Max/MSP file.

Knoll, J. Corey. "5 Screaming Amps." 2011. TS.

Montandon, Susannah. Message to the author. 27 May 2011. E-mail.

Pathak, Jhayson. Message to the author. 27 May 2011. E-mail.

Smallwood, Scott, et al. "Composing for Laptop Orchestra." Computer Music Journal 32 (2008): 9-25. Print.

Trueman, Daniel, et al. "Plork: The Princeton Laptop Orchestra, Year 1." Princeton University, 2006. Web. 17 May 2011.

Wolek, Nathan. "Improvising Laptop Ensemble Survey." Message to the author. 3 May 2011. E-mail. 